Nota Científica

\title{
Dieta invernal del cormorán imperial Phalacrocorax atriceps (Aves: Suliformes) en Bahía Caulín, Chiloé, sur de Chile
}

Winter diet of the imperial cormorant (Phalacrocorax atriceps) (Aves: Suliformes) in Caulín Bay, Chiloé, southern Chile

\section{Claudio N. Tobar ${ }^{1,2^{*}}$, David Carmona ${ }^{2}$, Jaime R. Rau ${ }^{2}$, Jaime A. Cursach ${ }^{2,3}$ y Jonnathan Vilugrón ${ }^{2}$}

\author{
'Departamento de Ciencias Básicas, Universidad Santo Tomás, Los Carrera 753, Osorno, Chile \\ ${ }^{2}$ Laboratorio de Ecología, Departamento de Ciencias Biológicas y Biodiversidad \& Programa IBAM, Universidad de Los Lagos, Casilla \\ 933, Osorno, Chile \\ ${ }^{3}$ ATLAS. Programa de Investigación Interdisciplinaria en Complejidad Territorial y Sustentabilidad, Departamento de Ciencias Sociales, \\ Universidad de Los Lagos, Casilla 933, Osorno, Chile \\ *Autor corresponsal: claudiobiomar@gmail.com
}

\begin{abstract}
The imperial cormorant (Phalacrocorax atriceps) is one of the most abundant cormorant species in the Chilean channels and fjords. Although its reproductive and non-reproductive distributions have been studied, works on its diet are inexistent. This paper describes the diet and determines the dominance and diversity of the prey consumed during the winter periods of 2011 and 2014 in Caulín Bay, Chiloé, southern Chile. A total of 73 pellets were collected (30 in 2011 and 43 in 2014). Prey were identified and classified into three categories: fishes (53.57\%), cephalopods (39.29\%) and crustaceans (7.14\%). When prey consumption was analyzed according to their distribution in the water column, we observed statistically significant differences in a preference of benthic demersal over pelagic prey.
\end{abstract}

Key words: Diet, imperial cormorant, seabirds, piscivorous

\section{INTRODUCCIÓN}

Los cormoranes son aves cosmopolitas piscívoras que frecuentan aguas interiores y costeras, desde regiones tropicales a polares, con un total de 39 especies que conforman el género Phalacrocorax (Nelson 2005). El éxito de este grupo está atribuido a su eficiencia como depredadores acuáticos (Gremillet et al. 2005), en relación con sus capacidades de natación y buceo (Gremillet 1997). El cormorán imperial Phalacrocorax atriceps es un ave marina voladora y buceadora asociada a mares fríos y endémica del sur de Sudamérica (Behn et al. 1955). En Chile, su distribución es desde la región del Biobío $\left(37^{\circ} \mathrm{S}\right)$ hasta Tierra del Fuego (ca., 52º S) (Araya \& Millie 1996). El cormorán imperial es una de las especies más numerosas de cormoranes dentro de los canales y fiordos chilenos (Jehl 1973, Imberti 2005, Garay et al. 2008), y la cuarta más abundante de las aves marinas de la Patagonia argentina (Frere et al. 2005). En cuanto a su ecología trófica es un predador tope con plasticidad en sus hábitos alimenticios ya que consume presas bentónicas, demersales y pelágicas, cuya contribución varía según el sitio y la etapa del ciclo reproductivo (Yorio et al. 2010, Ibarra et al. 2018). Estas características conllevan a estimar que las poblaciones de cormoranes imperiales puedan ser buenos indicadores de la presencia del stock de peces (Michalik et al. 2010), ya que éstos son el componente principal de su dieta (Malacalza et al. 1994, Ferrari et al. 2004, Bulgarella et al. 2008). Por otro lado, en Argentina es una especie relativamente bien estudiada, especialmente sobre aspectos de su distribución (Frere et al. 2005, Frixione 2010), reproducción (Malacalza \& Navas 1996, Arrighi \& Navarro 1998, Punta et al. 2003a, Svagelj \& Quintana 2011) y alimentación (Malacalza et al. 1994, Ferrari et al. 2004, Punta et al. 2003b, Bulgarella et al. 2008), sin embargo, en Chile, solo se ha estudiado su distribución reproductiva (Cursach et al. 2010, Kusch \& Marín 2013) y no-reproductiva (Kusch et al. 2007), sin antecedentes publicados sobre su dieta. Por lo tanto, los objetivos del presente trabajo fueron describir la dieta del cormorán imperial, determinando la importancia de las presas consumidas, y evaluar el consumo relativo de presas bentónica-demersales versus presas pelágicas durante los periodos invernales de 2011 y 2014 en la Bahía de Caulín, Chiloé, sur de Chile. 


\section{MATERIALES Y MÉTODOS}

Durante julio y agosto de 2011 y 2014 (inviernos australes) se recolectó un total de 73 egagrópilas del cormorán imperial (30 en 2011 y 43 en 2014) en un sitio de descanso ubicado en la isla Lacaos, ubicada en el humedal marino de bahía Caulín, en la isla grande de Chiloé, sur de Chile (41 ${ }^{\circ} 48^{\prime} \mathrm{S}$; $73^{\circ} 37^{\prime}$ O; ver descripción en Tobar et al. 2014) (Fig. 1). Las egagrópilas fueron colectadas durante la marea baja debido a la inaccesibilidad del lugar de muestreo durante pleamar. Cada egagrópila correspondió a lo regurgitado por un individuo adulto, siendo observados con un telescopio (60 x), durante el proceso de descanso y posterior regurgitación. Las egagrópilas fueron disgregadas en húmedo a través de un chorro constante de agua y los restos retenidos en un tamiz de $0,5 \mathrm{~mm}$ de abertura (Naya et al. 2000), posteriormente los restos duros contenidos fueron examinados bajo lupa estereoscópica WildM3 Heerbrugg (Wild Heerbrugg(C) a una magnificación de $10 \mathrm{X} / 23$, identificando los diferentes tipos de presas a partir de las partes duras de los peces (otolitos sagitta y huesos), cefalópodos (mandíbulas), crustáceos (restos de tegumento) y cualquier otro elemento que permitiera la caracterización de las presas consumidas
(Ferrari et al. 2004, Millones et al. 2005). La clasificación de los diferentes ítems alimenticios se realizó hasta el menor nivel taxonómico posible. Para ello se utilizó literatura de referencia y diversas claves de las potenciales presas del cormorán imperial (Falabella et al. 1995, Naveda 2001, Zagal \& Hermosilla 2008, Xavier \& Cherel 2009). Para cuantificar la importancia de las presas encontradas en las muestras según su distribución en la columna de agua (bentónica-demersal o pelágica), se utilizaron como estimadores la frecuencia de aparición $(\mathrm{Fa})$ y la frecuencia de ocurrencia (F\%) de cada especie en las egagrópilas. $\mathrm{Fa}=\left(\mathrm{fi} / \sum \mathrm{fi}\right) \times 100$, en donde $\mathrm{fi}=$ es el número de veces en las que aparece la especie presa i y $\sum \mathrm{fi}=$ número total de ocurrencias. Con esta fórmula se obtiene la frecuencia de aparición de cada especie presa en relación con el número total de ocurrencias (Maehr \& Brady 1986), mientras que la frecuencia de ocurrencia ( $\mathrm{F} \%$ ) es el porcentaje de muestras en las cuales está presente un determinado ítem presa sobre el número total de muestras estudiadas. Posteriormente, se utilizó la prueba de $\chi^{2}$ para bondad de ajuste y determinar si hubo diferencias dietaria de las frecuencias de ocurrencia de especies-presas consumidas según su distribución en la

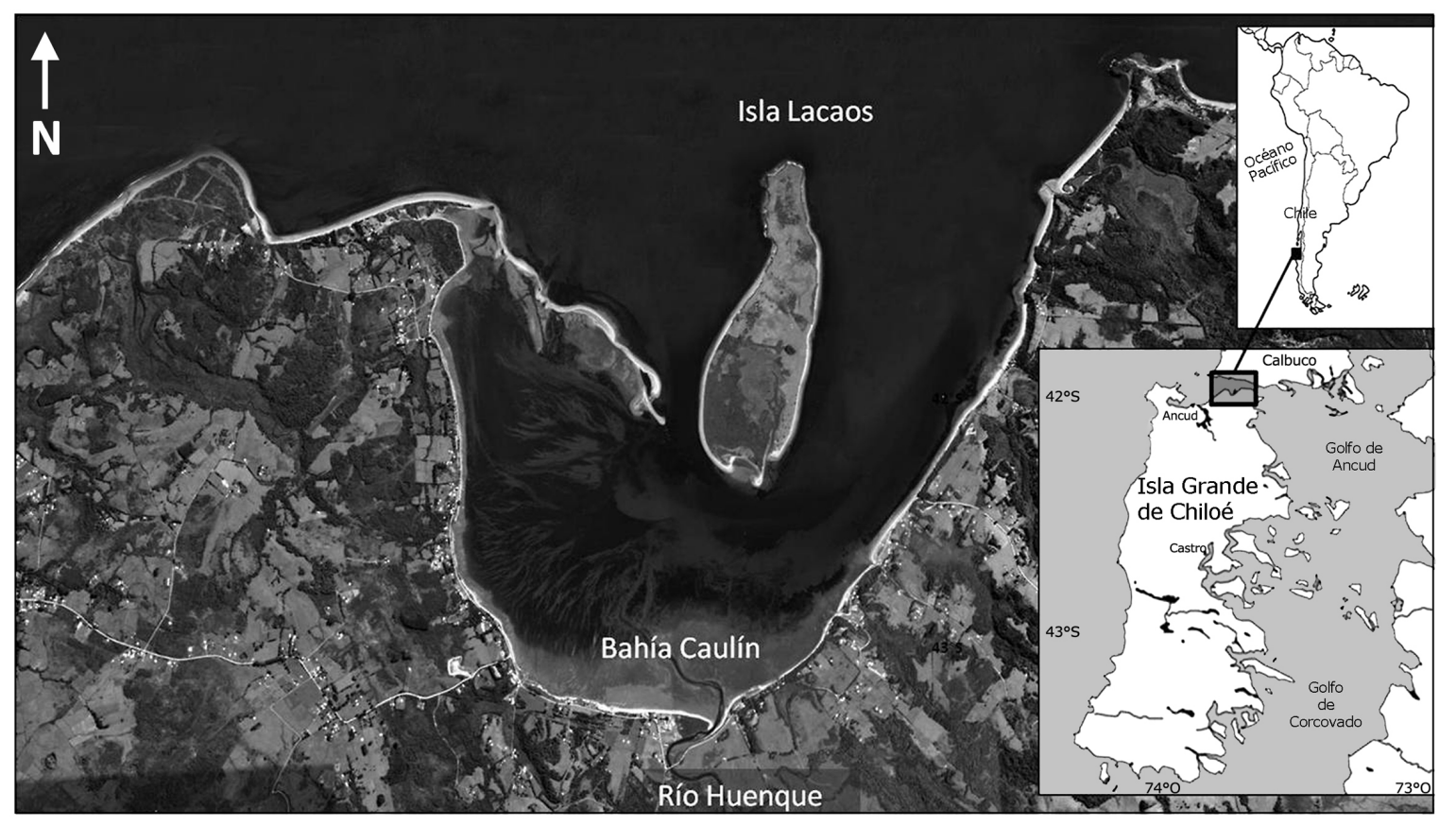

Figura. 1. Área de estudio, Isla Lacaos en bahía de Caulín (4149'S; $\left.73^{\circ} 38^{\prime} \mathrm{O}\right)$, Isla Grande de Chiloé, sur de Chile / Study area, Isla Lacaos in Caulín Bay $\left(41^{\circ} 49^{\prime} \mathrm{S}, 73^{\circ} 38^{\prime} \mathrm{W}\right)$, Chiloé Island, southern Chile 
columna de agua (presas pelágicas vs presas bentónicasdemersales), a través del paquete computacional estadístico en línea VassarStats: Website for Statistical Computation (CRichard Lowry 1998-2017)1.

\section{RESULTADOS Y DISCUSIÓN}

Tres categorías de presas se identificaron en la dieta del cormorán imperial las cuales correspondieron, de acuerdo con la frecuencia de aparición, a peces $(53,57 \%)$, cefalópodos $(39,29 \%)$ y crustáceos $(7,14 \%)$ (Tabla 1). En cuanto al ítem peces se identificaron 10 presas diferentes, de las cuales 7 fueron identificadas a nivel de especie; el róbalo Eleginops maclovinus (Cuvier, 1830), el mote Normanichthys crockeri (Clark, 1937), el pejerrey de mar Odontesthes regia (Humboldt, 1821), el lorcho Patagonotothen tessellata (Richardson, 1845), el doradito Paranotothenia magellanica (Forster, 1801), la borrachilla Scartichthys viridis (Valenciennes, 1836) y la cabrilla Sebastes oculatus (Valenciennes, 1833). Las otras tres presas se identificaron solo a nivel de género (Patagonotothen) y familia (Merlucciidae), y otra como indeterminada. En cuanto a los cefalópodos se identificaron al menos dos especies, el pulpo del sur Enteroctopus megalocyathus (Gould, 1852) y Octopus sp. Mientras que para los crustáceos solo se pudo identificar presas a nivel del subfilo Crustacea. Al comparar el consumo de presas según su distribución en la columna de agua (presas pelágicas $v s$ presas bentónicas-demersales) (Tabla 1), se observaron diferencias estadísticamente significativas $\left(\chi^{2}=81,56\right.$, g.l $1=$ $1, P<0,0001)$. Estos resultados revelaron que en la dieta invernal del cormorán imperial predominó el consumo de presas bentónica-demersales por sobre las pelágicas, lo cual se reflejó en la composición de presas de la dieta (Tabla 1).

Los resultados de este estudio determinaron que el cormorán imperial durante el invierno consume peces, cefalópodos y crustáceos, siendo el ítem peces el que registró la mayor frecuencia de ocurrencia en la dieta con un $82,19 \%$, destacando E. maclovinus como la presa más consumida de hábito bentónica-demersal (Tabla 1), lo que confirma su caracterización como una especie piscívora, seguido por cefalópodos representados por Octopus sp y Enteroctopus megalocyathus. Diversos autores señalan que el cormorán imperial consume principalmente peces durante la época estival (Malacalza et al. 1994, Bulgarella et al. 2008, Yorio et al. 2010, 2017; Ibarra et al. 2018) y secundariamente cefalópodos, mientras que en este trabajo se destaca la importancia de los cefalópodos en la dieta invernal del cormorán imperial, con una frecuencia de ocurrencia del 60,27\%, siendo Octopus sp. la presa más importante en la dieta del cormorán imperial (Tabla 1). Del ítem crustáceos, en este trabajo no fue posible identificar ninguna especie debido al mal estado de las estructuras y la

Tabla 1. Frecuencia de aparición (Fa\%) y frecuencia de ocurrencia (F\%) de presas en la dieta del $\boldsymbol{P}$. atriceps de la Isla Lacao, Bahía de Caulín, Chiloé / Appearance frequency (Fa\%) and frequency of occurrence (F\%) of prey in the diet of $P$. atriceps of Isla Lacao, Caulín Bay, Chiloé

\begin{tabular}{lrrl}
\hline \multicolumn{1}{c}{ Ítem presas } & $\mathrm{Fa} \%$ & $\mathrm{~F} \%$ & \multicolumn{1}{c}{ Hábitos/Presa } \\
\hline Peces & & & \\
Eleginops maclovinus & 19,64 & 30,14 & Bentónico-demersal \\
Odontesthes regia & 8,04 & 12,33 & Pelágico \\
Scartichthys viridis & 5,36 & 9,59 & Bentónico-demersal \\
Paranotothenia magellanica & 4,46 & 8,22 & Bentónico-demersal \\
Patagonotothen tessellata & 3,57 & 6,85 & Bentónico-demersal \\
Sebastes oculatus & 2,68 & 5,48 & Bentónico-demersal \\
Normanichthys crockeri & 1,79 & 4,1 & Pelágico \\
Patagonotothen sp. & 0,89 & 2,74 & Bentónico-demersal \\
Merlucciidae & 0,89 & 1,37 & Bentónico-demersal \\
Peces indeterminados & 6,25 & 1,37 & Indeterminado \\
Total & 53,57 & 82,19 & \\
Cefalópodos & & & \\
Enteroctopus megalocyathus & 8,04 & 12,33 & Bentónico-demersal \\
Octopus sp. & 31,25 & 47,94 & Bentónico-demersal \\
Total & 39,29 & 60,27 & \\
Crustáceos & & & \\
Crustáceos indeterminados & 7,14 & 10,95 & Indeterminado \\
\hline
\end{tabular}

${ }^{1}<$ http://vassarstats.net/> 
erosión provocada por los jugos gástricos de los cormoranes imperiales en su proceso de digestión, representando el $7,14 \%$ del total de la dieta y una frecuencia de ocurrencia del 10,95\%. Punta et al. (1993) señala que, durante el periodo pre postura del cormorán, hay una mayor ocurrencia de crustáceos que peces mientras, Malacalza et al. (1994) destacan el aumento del consumo de cefalópodos durante la etapa de crianza de los polluelos del cormorán.

Respecto al predominio en el consumo de presas bentónica-demersales por sobre las pelágicas, los resultados observados en este estudio, coinciden con trabajos realizados en Argentina durante la temporada reproductiva, en los cuales los peces consumidos en mayor frecuencia por el cormorán imperial fueron de hábitos bentónico-demersales, siendo el congrio (Raneya fluminensis) y la anchoíta (Engraulis anchoita) las principales especie-presa bentónica y pelágica en la dieta del cormorán imperial (Malacalza et al. 1994, Ferrari et al. 2004, Harris et al. 2016).

La mayor abundancia de E. maclovinus en la dieta del cormorán imperial podría reflejar una mayor disponibilidad de este recurso en el área de estudio, lo cual concordaría con los registros de Pequeño \& Lamilla (1995) donde el róbalo fue uno de los peces más abundantes en los canales de Chiloé. De acuerdo con Harris (2014) la especialización en un tipo de presa particular por parte de los cormoranes imperiales podría evidenciar una disponibilidad de recursos estable a largo plazo, es por ello que se deben realizar investigaciones en el área de estudio que comparen el espectro trófico a lo largo de su ciclo reproductivo y en las diferentes estaciones del año, a fin de contar con mayor información biológica sobre Phalacrocorax atriceps, presente en el sur de la Patagonia chilena continental.

\section{Agradecimientos}

A Ramón Molina y familia (Ostras Caulín) por el apoyo logístico y traspaso de conocimiento sobre las aves presentes en Caulín, al Dr. Christopher B. Anderson por las correcciones al manuscrito. Finalmente se agradece a los Núcleos de Investigación BIODES y BIODES 2.0 de la Universidad de Los Lagos.

\section{LITERATURA CITADA}

Araya BM \& GH Millie. 1996. Guía de campo de las aves de Chile, 389 pp. Editorial Universitaria, Santiago.

Arrighi AC \& JL Navarro. 1998. Ecología reproductiva del Cormorán Imperial (Phalacrocorax atriceps) en Isla Deseada (Santa Cruz, Argentina). Hornero 15: 64-67.

Behn F, J Goodall, A Johnson \& R Philippi. 1955. The geographic distribution of Blue-eyed shags, Phalacrocorax albiventer and Phalacrocorax atriceps. Auk 72: 6-13.

Bulgarella M, LC Pizarro, F Quintana, A Sapoznikow, A Gosztonyi \& L Kuba. 2008. Diet of imperial cormorants (Phalacrocorax atriceps) and rock shags (P. magellanicus) breeding sympatrically in Patagonia, Argentina. Ornitología Neotropical 19: 553-563.
Cursach JA, A Simeone, R Matus, O Soto, R Schlatter, C Tobar \& J Ojeda. 2010. Distribución reproductiva del cormorán imperial (Phalacrocorax atriceps) en Chile. Boletín Chileno de Ornitología 16: 9-16.

Falabella F, R Meléndez \& M Vargas. 1995. Claves osteológicas para peces de Chile central. Un enfoque arqueológico, 208 pp. Artegrama, Santiago.

Ferrari S, B Alegre \& P Gandini. 2004. Dieta del cormorán imperial (Phalacrocorax atriceps) en el sur de Santa Cruz (Patagonia, Argentina). Ornitología Neotropical 15: 103-110.

Frere E, F Quintana \& P Gandini. 2005. Cormoranes de la costa patagónica: estado poblacional, ecología y conservación. Hornero 20: 35-52.

Frixione MG. 2010. El Cormorán Imperial (Phalacrocorax atriceps) en el lago Nahuel Huapi: Distribución, abundancia y amenazas potenciales de aves carroñeras. Hornero 25(2): 61-65.

Garay G, O Guineo, E Mutschke \& C Ríos. 2008. Tamaño, estructura y distribución estacional de poblaciones de aves acuáticas en el Fiordo Última Esperanza y Canal Señoret, Región de Magallanes. Anales del Instituto de la Patagonia 36: 33-44.

Gremillet D. 1997. Catch per unit effort, foraging efficiency, and parental investment in breeding great cormorants (Phalacrocorax carbo carbo). ICES Journal Marine Science 54: 635-644.

Gremillet D, G Kuntz, AJ Woakes, C Gilbert, JP Robin, Y Le Maho \& PJ Butler. 2005. Year-round recordings of behavioural and physiological parameters reveal the survival strategy of a poorly insulated diving endotherm during the Arctic winter. Journal of Experimental Biology 208(22): $4231-4241$

Harris SE. 2014. Comportamiento de búsqueda de alimento del Cormorán Imperial (Phalacrocorax atriceps): caracterización, consistencias individuales y factores determinantes. Tesis Doctoral, Facultad de Ciencias Exactas y Naturales, Universidad de Buenos Aires, Buenos Aires, 140 pp. <http://digital.bl.fcen.uba.ar/Download/Tesis/ Tesis_5496_Harris.pdf $>$

Harris S, F Quintana, J Ciancio, L Riccialdelli \& A RayaRey. 2016. Linking foraging behavior and diet in a diving seabird. Marine Ecology 37: 419-432.

Ibarra C, C Marinao, N Suárez \& P Yorio. 2018. Differences between colonies and chick-rearing stages in Imperial Cormorant (Phalacrocorax atriceps) diet composition: Implications for trophic studies and monitoring. The Wilson Journal of Ornithology 130: 224-234.

Imberti S. 2005. Distribución otoñal de aves marinas y terrestres en los canales chilenos. Anales del Instituto de la Patagonia 33: $21-30$.

Jehl JR. 1973. The distribution of marine birds in Chilean waters in winter. Auk 90: 114-135.

Kusch A \& M Marín. 2013. Distribución de sitios reproductivos de cormoranes Phalacrocorax spp. (Pelecaniformes) en el Estrecho de Magallanes y costas hacia el sur $\left(52^{\circ}-56^{\circ} \mathrm{S}\right)$, Chile. Anales del Instituto de la Patagonia 41: 131-139.

Kusch A, M Marín, D Oheler \& S Drieschman. 2007. Notas sobre la avifauna de Isla Noir $\left(54^{\circ} 28^{\prime} \mathrm{S}-73^{\circ} 00^{\prime} \mathrm{W}\right)$. Anales del Instituto de la Patagonia 35(2): 61-66. 
Maehr DS \& JR Brady. 1986. Food habits of bobcats in Florida. Journal of Mammalogy 67(1): 133-138.

Malacalza VE \& JR Navas. 1996. Biología y ecología reproductiva de Phalacrocorax albiventer (Aves: Phalacrocoracidae) en Punta León, Chubut, Argentina. Ornitología Neotropical 7: 53-61.

Malacalza VE, TI Poretti \& NM Bertellotti. 1994. La dieta de Phalacrocorax albiventer en Punta León (Chubut, Argentina) durante la temporada reproductiva. Ornitología Neotropical 5: 91-97.

Michalik A, HJ van Noordwijk, P Brickle, T Eggers \& P Quillfeldt. 2010. The diet of the Imperial Shag Phalacrocorax atriceps at a colony on New Island, Falkland/ Malvinas Islands combining different sampling techniques. Polar Biology 33(11): 1537-1546

Millones A, E Frere \& P Gandini. 2005. Dieta del cormorán gris (Phalacrocorax gaimardi) en la ría Deseado, Santa Cruz, Argentina. Ornitología Neotropical 16: 519-527.

Naveda I G-G. 2001. Patrones morfológicos del otolito sagitta de algunos peces óseos del mar peruano. Boletín del Instituto del Mar del Perú 20(1/2): 4-83.

Naya DE, R Vargas \& M Arim. 2000. Análisis preliminar de la dieta del león marino del sur (Otaria flavescens) en Isla de Lobos, Uruguay. Boletín de la Sociedad Zoológica del Uruguay 12: 14-21.

Nelson JB. 2005. Pelicans, cormorants, and their relatives: The Pelecaniformes, 680 pp. Oxford University Press, Oxford.

Pequeño G \& J Lamilla. 1995. Peces intermareales de la costa de Llanquihue (Chile): Composición taxonómica, abundancia relativa y gradiente de distribución longitudinal. Revista de Biología Marina 30(1): 7-27.

Punta GE, JRC Saravia \& PM Yorio. 1993. The diet and foraging behaviour of two Patagonian cormorants. Marine Ornithology 21: 27-36.
Punta G, P Yorio, G Herrera \& J Saravia. 2003a. Biología reproductiva de los cormoranes Imperial (Phalacrocorax atriceps) y Cuello Negro (P. magellanicus) en el Golfo San Jorge, Chubut, Argentina. El Hornero 18: 103-111.

Punta G, P Yorio \& G Herrera. 2003b. Temporal patterns in the diet and food partitioning in Imperial Cormorants (Phalacrocorax atriceps) and Rock Shags (P. magellanicus) breeding at Bahía Bustamante, Argentina. Wilson Bulletin 115: 308-316.

Svagelj WS \& F Quintana. 2011. Breeding performance of the Imperial Shag (Phalacrocorax atriceps) in relation to year, laying date and nest location. Emu 111: 162-165.

Tobar C, J Rau, N Fuentes, A Gantz, J Cursach, CG Suazo, A Santibáñez \& J Pérez-Shultheiss. 2014. Diet of the Chilean flamingo Phoenicopterus chilensis (Phoenicopteriformes: Phoenicopteridae) in a coastal wetland in Chiloé, Southern Chile. Revista Chilena de Historia Natural 87:15. < doi: 10.1186/s40693-014-0015-1>

Xavier JC \& Y Cherel. 2009. Cephalopod beak guide for the Southern Ocean, 129 pp. British Antarctic Survey, Cambridge.

Yorio P, S Copello, L Kuba, A Gosztonyi \& F Quintana. 2010. Diet of imperial cormorants Phalacrocorax atriceps breeding at Central Patagonia, Argentina. Waterbirds 33(1): 70-78.

Yorio P, C Ibarra \& C Marinao. 2017. Induced regurgitation versus stomach sampling: assessing their value for the characterization of imperial cormorant (Phalacrocorax atriceps) diet. Waterbirds 40(2): 162-167.

Zagal C \& C Hermosilla. 2008. Guía de invertebrados marinos del sur de Chile, 264 pp. Editorial Fantástico Sur, Punta Arenas.

Recibido el 7 de diciembre 2018 y aceptado el 7 de mayo de 2019

Editor: Claudia Bustos / Colaborador editor: Pablo M. Yorio 